\title{
Caracterización de la Harina de Semillas de Amaranto Amaranthus Caudatus para Elaboración de Pan en Mezclas con Harina de Trigo
}

\author{
CONSUELO PÉREZ ${ }^{*}$, ÓSCAR LUZURIAGA' \\ 'Química de Alimentos, Facultad de Ciencias Químicas, Universidad Central del Ecuador \\ *Correspondencia: consuenov81@yahoo.com
}

\section{Resumen}

Amaranthus caudatus es un pseudocereal introducido en el Ecuador a partir de 1988 y ha demostrado adaptabilidad en suelos andinos, obteniéndose cultivos promisorios; sin embargo, los avances agronómicos desarrollados hasta la fecha no han sido suficientes para poner de manifiesto el cultivo frente a una población consumidora de productos elaborados. Considerando la cultura de alimentación ecuatoriana, la realización y estudio de harina de semilla de amaranto es una de las alternativas alimentarias a ser puesta en marcha, que junto a la introducción de producto de panificación son un aporte a la estimulación de dicho cultivo e indagación de posteriores investigaciones. Siendo el pan el principal representante de los productos de panificación y un producto alimenticio de primera necesidad, se evaluó a condiciones medioambientales la estabilidad de panes realizados con 5\%, 10\% y 15\% de harina de semilla de amaranto frente a un blanco (pan común). Los resultados obtenidos de las formulaciones evaluadas respecto al blanco demostraron no existir diferencia entre los tiempos de vida útil, así como tampoco en cuanto al sabor; lo que indica que dichas formulaciones pueden ser introducidas en el mercado sin mostrar variabilidad en cuanto a estos parámetros.

Palabras clave: Amaranto, pan - amaranto, análisis alimentos, conservación de alimentos, tecnología de alimentos.

\section{Abstract}

Amantanthus caudatus is a type of pseudocereal which has been used in Ecuador since 1988. It has show a good adaptability in the Andean region, at the same time obtaining promising crops. However, the latest agricultural achievements haven't been enough to place the mentioned crop in front of a consumer population of elaborated products. Considering the Ecuadorian food habits the realization, and studies of flour of the Amaranthus caudatus seed, it can be concluded that the flour is one of the best food supplies. Together with the introduction of bakery products these are reasons to encourage its cultivation. Talking about bread as the principal representative of baked goods and are of our needed food supplies, under the environmental conditions, its stability has been evaluated using $5 \%, 10 \%$ and $15 \%$ of the flour of Amaranthus caudatus compared to a while bread. The obtained results of the evaluated formulas considering the white bread, demonstrate no difference en the lasting time and flavor. It indicates that the mentioned formulas can be brought to the market without showing variability en these parameters.

Key words: Amaranto, amaranto - bread, food analysis, food conservation, technology of foods. 


\section{Introducción}

Las características climatológicas que tiene la Sierra ecuatoriana son fundamentales para el cultivo y evaluación de potenciales especies promisorias introducidas como Amaranthus caudatus, que ha sido reconocida por organismos internacionales, como la FAO y UNICEF, comprometidos en el mejoramiento nutricional de las poblaciones de escasos recursos económicos de países en vías de desarrollo, asimismo la NASA interesada en la obtención de productos que garanticen la alimentación de las tripulaciones en el espacio, han brindado su contingente para evaluar a tan preciado pseudocereal dentro de diferentes ámbitos.

Dentro de las variedades de amaranto existentes a nivel del mundo entero, Amaranthus caudatus corresponde a una de las especies productoras de grano, que ha logrado reconocimiento en base a sus características, contenidos de proteína, almidón y grasa presentes en la semilla.

En países como México, Bolivia y Perú el amaranto mantiene equidad de consumo frente a los cereales; en Ecuador, Amaranthus caudatus es una especie introducida cuyas primeras evaluaciones se emprenden en 1988, lográndose semillas agrícolamente adaptadas, a partir de las que se han incentivado planes de cultivo en localidades andinas.

En la actualidad, el interés de pequeñas masas productoras radica en la exportación del grano para introducirlo en formulaciones de productos extrusados como ingrediente en cereales para desayuno; la característica de reventar como palomita de maíz con ligero sabor a nuez es otro de sus llamativos para integrarlo a productos afines a los anteriores; la utilización en embutidos como texturizadores, son las aplicaciones que más han llamado la atención; lograr novedosas presentaciones de frutas confitadas recubiertas con almidón de amaranto, así como también la utilización en pastas frías son otras de las aplicaciones que se han otorgado al almidón a nivel internacional.

La presente investigación anhela contribuir al conocimiento, difusión y evaluación del amaranto presente en el país para lograr el desarrollo de productos que probablemente podrían formar parte de la dieta ecuatoriana.
Si bien, la harina de amaranto es factible de ser utilizada individualmente en preparaciones culinarias caseras, los derivados de cereales, cuyo principal representante es el pan, cumple con la característica de ser un alimento de primera necesidad, que sólo es factible de ser realizado con harinas de trigo o centeno.

La sustitución de porciones de estas harinas por harina de semilla de amaranto en la elaboración de pan común serían factibles de ser evaluadas, física, química y sensorialmente en los productos elaborados; no obstante, estos parámetros se han enfocado hacia el estudio de estabilidad de los mismos con la finalidad de que el presente aporte sea un preámbulo hacia estudios químicos y nutricionales más profundos en caso de ser meritorios.

\section{Materiales y Métodos}

\section{Determinación del tamaño de la semilla}

Método: Métrico

\section{Materiales:}

Tornillo calibrador

semilla de Amaranthus caudatus

$$
\bar{X}=\frac{\sum X i}{N i}
$$

Semillas de Amaranthus caudatus

$\bar{X}=$ Diámetro medio de la semilla

Xi= Diámetro individual de cada semilla medida

$\mathrm{Ni}=$ Número total de semillas medidas

\section{Análisis granulométrico}

A un molino CORONA se le redujo el tamaño de las estrías, se acoplaron sistemas de poleas y eléctrico para la optimización del trabajo.

Método: Tamización

Materiales y equipos:

Serie de tamices

Motor vibrador (Ro - Tap)

Balanza sensible a $100 \mathrm{mg}$

Espátula

Escobilla de cerdas suaves 


\section{Diámetro final de la partícula}

$$
D=\frac{\sum X i / d p^{2}}{\sum X i / d p^{3}}
$$

$\mathrm{D}=$ Diámetro final de la partícula, $\mathrm{mm}$

$X i=$ Fracción en peso retenida en cada tamiz $\mathrm{g} / \mathrm{g}$

$d p=$ Diámetro medio de tamices en $\mathrm{cm}$

\section{Determinación de pH}

Método: Potenciométrico

\section{Materiales y Equipos:}

Potenciómetro

Soluciones buffer de $\mathrm{pH}(7,4,10)$

Vaso de precipitación de $250 \mathrm{ml}$

Solución de $\mathrm{KCl} 3 \mathrm{M}$

Papel absorbente delicado

Piceta

Agua destilada

\section{Determinación de Acidez Titulable}

Método: Volumétrico

\section{Materiales y Equipos:}

Erlenmeyers de $50 \mathrm{ml}$ y $250 \mathrm{ml}$.

Pipeta volumétrica de $10 \mathrm{ml}$

Bureta de $25 \mathrm{ml}$

Hidróxido de sodio $0,02 \mathrm{~N}$

Solución de fenolftaleína 0,1\%

Alcohol etílico de $90 \%$ neutralizado

Papel filtro de poro amplio

$$
A=\frac{490 N V}{m(100-h)} \times \frac{V_{1}}{V_{2}}
$$

$A=$ contenido de acidez expresado en porcentaje de masa de ácido sulfúrico

$N=$ normalidad de la solución de hidróxido de sodio

$V=$ volumen de la solución de hidróxido de sodio empleado en la titulación, $\mathrm{ml}$

$V_{1}=$ Volumen de alcohol empleado, $\mathrm{ml}$

$V_{2}=$ Volumen de la alícuota, $\mathrm{ml}$

$m=$ masa de la muestra, $g$

$h=$ porcentaje de humedad en la muestra

\section{Determinación de humedad}

Método: Gravimétrico

Materiales y Equipos:

Desecador con deshidratante

Pinza para cápsulas

Cápsulas de aluminio de fondo plano de $6 \mathrm{~cm}$ de diámetro y $1,5 \mathrm{~cm}$ de profundidad

Estufa

Balanza analítica son sensibilidad a 0,1 mg

$$
P c=\frac{m_{2}-m_{3}}{m_{2}-m_{1}} x 100
$$

$P c=$ Perdida por calentamiento, en porcentaje de masa.

$\mathrm{m}_{1}=$ masa de la cápsula de aluminio tarada, $\mathrm{g}$

$m_{2}=$ masa de la cápsula de aluminio con la muestra sin secar, $\mathrm{g}$

$m_{3}=$ masa de la cápsula de aluminio con la muestra seca, $\mathrm{g}$

\section{Determinación de grasa bruta}

Método: Hidrólisis ácida - Soxhlet

\section{Reactivos}

Ácido clorhídrico concentrado

Éter de petróleo

\section{Materiales y Equipos:}

Erlenmeyer de $500 \mathrm{ml}$

Papel filtro de poro amplio

Embudo

Agua caliente

Estufa

Cajas de aluminio

Algodón (libre de grasa)

Equipo automático de extracción Soxhlet

Sorbona

Vasos de extracción adaptables al equipo, previamente tarados

Pinza para vasos

Desecador provisto con deshidratante adecuado

$$
G=\frac{m_{2}-m_{1}}{m_{3}} \times 100
$$

$G=$ contenido de grasa en porcentaje de masa

$m_{l}=$ masa del vaso tarado, $g$

$m_{2}=$ masa del vaso con grasa, $g$

$m_{3}=$ masa de muestra seca, $\mathrm{g}$ 


\section{Determinación de fibra bruta}

Método: Digestión ácido - básica (Método WEENDE)

\section{Materiales y Equipos:}

Papel filtro cualitativo

Papel filtro cuantitativo

Embudos de vidrio

Matraces erlenmeyers $500 \mathrm{ml}$

Guante de calor

Agua destilada

Acido sulfúrico $1,25 \% \mathrm{v} / \mathrm{v}$

Hidróxido de sodio $1,25 \% \mathrm{p} / \mathrm{v}$

Cocineta eléctrica

Balanza analítica sensible al $0,1 \mathrm{mg}$

Desecador provisto con deshidratante adecuado

Núcleos de ebullición

Pinza para erlenmeyers

$$
F=\frac{p_{2}-p_{1}}{m} x 100
$$

$F=$ contenido de fibra en porcentaje de masa

$P_{7}=$ masa del papel filtro libre de humedad, $\mathrm{g}$

$P_{2}=$ masa del papel filtro con la fibra, $g$

$m=$ masa de muestra, $g$

\section{Determinación de cenizas}

\section{Método: Gravimétrico}

\section{Materiales y Equipos:}

Mufla ajustada a $550^{\circ} \mathrm{C}$

Crisoles de porcelana

Pinza para crisoles

Desecador provisto con deshidratante adecuado

Cocineta eléctrica

Espátula

Balanza analítica al 0,1 mg

$$
P c=\frac{m_{2}-m_{3}}{m_{2}-m_{1}} \times 100
$$

$P_{c}=$ Porcentaje de ceniza

$m_{1}=$ masa del crisol tarado, $\mathrm{g}$

$m_{2}=$ masa del crisol con la muestra sin secar, $g$

$m_{3}=$ masa del crisol con la muestra seca, $g$

\section{Determinación de proteína bruta}

Método Kjeldahl

Materiales y Equipos:

Unidad de digestión y destilación Kieldahl.

Balones Kjeldahl

Núcleos de ebullición

Papel celofán, libre de nitrógeno

Catalizador Kieldahl

Ácido sulfúrico concentrado

Agua destilada

Probeta graduada con capacidad para $10 \mathrm{ml}$

Ácido bórico con indicador mezcla (rojo de metiloverde de bromocresol)

Matraz volumétrico de $250 \mathrm{ml}$

Hidróxido de sodio $50 \% \mathrm{p} / \mathrm{v}$.

Ácido clorhídrico/ sulfúrico $0,1 \mathrm{~N}$

Bureta automática

$$
\text { Proteina bruta }=\frac{\text { NxVxpmeqx6.25 }}{m} \times 100
$$

$N=$ Normalidad del ácido

$V=$ Volumen de ácido utilizado en la titulación, $\mathrm{ml}$ pmeq $=$ peso mili equivalente del Nitrógeno

6,25 = factor para conversión de proteína

$m=$ gramos de muestra

\section{Determinación de aminoácidos}

Método Cromatografía Líquida de Alta Presión HPLC (INIAP)

\section{Materiales y Equipos:}

Balanza analítica con sensibilidad al 0,1 mg

Microespátula

Viales para digestión de muestra

Gradilla metálica

Estufa regulable

Rotavapor

Membranas para filtración de 0,45 mesh

Equipo para análisis de aminoácidos Shimadzu modelo

$L C-10 A D$

Columna Shim - pack AMINO - Na

\section{Reactivos:}

$\mathrm{HCl} 6 \mathrm{~N}$

Solución reguladora de $\mathrm{pH} \mathrm{2,2}$ 


\section{Determinación de triptófano}

Método Colorimétrico

\section{Materiales y Equipos:}

Balanza analítica con sensibilidad al 0,1 mg

Estufa incubadora

Centrífuga

Pipetas volumétricas

Gradilla para tubos de ensayo

Tubos de ensayo de $13 \times 100 \mathrm{~mm}$ con tapón de rosca.

Tubos de ensayo de $16 \times 150 \mathrm{~mm}$

Tubos para colorímetro

Espectrofotómetro

\section{Reactivos:}

Solución ferroacética

Solución de papaína

Solución estándar de triptófano (100 mg/l)

\section{Azúcares reductores}

Método: Volumétrico (LUFF - SCHOORL)

\section{Materiales y reactivos:}

Cocineta eléctrica

Equipo de reflujo

Núcleos de ebullición

Pipeta volumétrica de $10 \mathrm{ml}$.

Pipeta volumétrica de $25 \mathrm{ml}$.

Balones aforados de $100 \mathrm{~mL}$

Agua destilada

Solución de hidróxido de sodio $35 \% \mathrm{p} / \mathrm{v}$

Ácido sulfúrico 3M

$\mathrm{KI}(\mathrm{s})$

Papel filtro

Soluciones de Carrez I y II

Solución de $\mathrm{Na}_{2} \mathrm{~S}_{2} \mathrm{O}_{3} 0,1 \mathrm{~N}$

Reactivo de Luff

Solución de almidón $1 \%$

Bureta

Pipetas graduadas

Papel filtro

$C(m l)=b-a v$

$b=$ consumo de la solución de $\mathrm{Na}_{2} \mathrm{~S}_{2} \mathrm{O}_{3} 0,1 \mathrm{~N}$ en el

blanco

av = consumo de la solución de $\mathrm{Na}_{2} \mathrm{~S}_{2} \mathrm{O}_{3}, 0,1 \mathrm{~N}$ en la muestra antes de la inversión

$\mathrm{C}=$ valor para obtener la cantidad de azucares totales según la tabla de equivalencia

\section{Determinación del contenido de almidón}

Método Polarimétrico

\section{Materiales y reactivos}

Balón volumétrico de $100 \mathrm{ml}$.

Baño de agua hirviente

Polarímetro

Tubo de polarímetro de $200 \mathrm{~mm}$

Balanza analítica con aprox. 0,1 mg

Piceta

Acido clorhídrico 1,124\%

Papel filtro

Termómetro

\section{Reactivos}

Solución de Carrez I

Solución de Carrez II

$$
\operatorname{Almidón}(\%)=\frac{100_{x} a_{x} 100}{[a]_{D_{x}} l_{x} 5}
$$

$\alpha=$ ángulo de desviación observado en el polarímetro

$[a]_{D}=$ rotación específica del almidón

I = longitud del tubo del polarímetro en decímetros

\section{Determinación de almidón como azúcares totales}

Método Volumétrico (LUFF - SCHOORL)

\section{Materiales y reactivos:}

Cocineta eléctrica

Equipo de reflujo

Núcleos de ebullición

Pipeta volumétrica de $1 \mathrm{ml}$

Pipeta volumétrica de $25 \mathrm{ml}$

Balones aforados de $100 \mathrm{ml}$

Baño de agua hirviente

Termómetro

Agua destilada

Ácido clorhídrico concentrado

Solución de hidróxido de sodio $35 \% \mathrm{p} / \mathrm{v}$

Acido sulfúrico $3 \mathrm{M}$

$\mathrm{KI}(\mathrm{s})$

Papel filtro

Soluciones de Carrez I y II

Solución de $\mathrm{Na}_{2} \mathrm{~S}_{2} \mathrm{O}_{3} \mathrm{O}, 1 \mathrm{~N}$

Reactivo de Luff

Solución de almidón 1\%

Bureta 
Pipetas graduadas de $5 \mathrm{ml}$

Papel filtro de poro amplio

$C(m l)=b-a$

$\mathrm{b}=$ consumo de la solución de $\mathrm{Na}_{2} \mathrm{~S}_{2} \mathrm{O}_{3}, 0,1 \mathrm{~N}$ en el blanco

$\mathrm{a}=$ consumo de la solución de $\mathrm{Na}_{2} \mathrm{~S}_{2} \mathrm{O}_{3} 0,1 \mathrm{~N}$ en la muestra

$\mathrm{C}=$ valor para obtener la cantidad de azúcares totales según la tabla de equivalencia

\section{Extracción de almidón}

Método Sedimentación

\section{Materiales y Equipos:}

Molino

Agua

Recipientes hondos que permitan la sedimentación

Recipientes planos que permitan la evaporación

Estufa regulada no mayor a $50^{\circ} \mathrm{C}$

Fundas plásticas

\section{Porcentaje de Amilosa}

\section{Método Colorimétrico}

\section{Materiales, Equipos y Reactivos:}

Balones aforados de $50 \mathrm{ml}$

Balones aforados de $100 \mathrm{~m}$

Balanza con aproximación al $0,1 \mathrm{mg}$

Espátula

Agua destilada

Piceta

Etanol $95 \% \mathrm{v} / \mathrm{v}$

Hidróxido de sodio $1 \mathrm{~N}$

Estándar de amilosa

Estándar de amilopectina

Hidróxido de sodio $0,09 \mathrm{~N}$

Acido clorhídrico $30 \% \mathrm{v} / \mathrm{v}$

Solución de yodo $2 \%$

Espectrofotómetro

\section{Amilograma}

Método Amilograma de cocción

\section{Materiales y Equipos:}

Balanza monoplano sensible al $100 \mathrm{mg}$

Espátula

Agua destilada

Amilógrafo Brabender con termorregulador y compresor Licuadora

\section{Análisis físico - químicos del pan}

Se reemplazó la harina fortificada para panificación por harina de amaranto en los siguientes porcentajes $5 \%, 10 \%$ y $15 \%$; se mantiene sin alteración los restantes ingredientes.

Las formulaciones a evaluarse de acuerdo a la maquinaria disponible para un Kilogramo de harina y sus equivalentes fueron las siguientes:

\section{Formulación del blanco}

\begin{tabular}{cc}
\hline MATERIA PRIMA & CANTIDAD, g \\
\hline Harina panadera fortificada & 1000 \\
Sal & 20 \\
Azúcar & 80 \\
Manteca & 250 \\
Agua & 550 \\
Levadura & 40 \\
\hline
\end{tabular}

2. Formulación al $5 \%$

\begin{tabular}{cc}
\hline MATERIA PRIMA & CANTIDAD, g \\
\hline Harina panadera fortificada & 950 \\
Harina de amaranto & 50 \\
Sal & 20 \\
Azúcar & 80 \\
Manteca & 250 \\
Agua & 550 \\
Levadura & 40 \\
\hline
\end{tabular}

3. Formulación al $10 \%$

\begin{tabular}{cc}
\hline MATERIA PRIMA & CANTIDAD, g \\
\hline Harina panadera fortificada & 900 \\
Harina de amaranto & 100 \\
Sal & 20 \\
Azúcar & 80 \\
Manteca & 250 \\
Agua & 550 \\
Levadura & 40 \\
\hline \multicolumn{2}{c}{ 4. Formulación al 15\% } \\
\hline MATERIA PRIMA & CANTIDAD, g \\
\hline Harina panadera fortificada & 850 \\
Harina de amaranto & 150 \\
Sal & 20 \\
Azúcar & 80 \\
Manteca & 250 \\
Agua & 550 \\
\hline Levadura & 40 \\
\hline
\end{tabular}




\section{Metodología}

Las determinaciones se llevaron a cabo durante cuatro semanas consecutivas en las que se realizaron cuatro horneadas correspondientes a los días lunes y se analizaron por cuatro días seguidos, es decir, hasta los días jueves. Se tomó en consideración la temperatura ambiental y la humedad relativa a la que se encontraban los panes en el laboratorio.

\section{Determinación de acidez}

Método: Volumétrico

\section{Materiales y Equipos:}

Vasos de precipitación $400 \mathrm{ml}$

Varilla de agitación

Espátula

Balanza analítica con sensibilidad al $0,1 \mathrm{mg}$

Pipeta graduada de $5 \mathrm{ml}$

Probeta graduada de $100 \mathrm{ml}$

Agua destilada hervida y fría

Embudos de vidrio

Papel filtro de poro amplio

Pipeta volumétrica de $25 \mathrm{ml}$

Balón aforado de $100 \mathrm{ml}$

Matraces erlenmeyer de $250 \mathrm{ml}$ de capacidad

Bureta 0,1 ml

Fenolftaleína 1\%

Hidróxido de sodio $0,02 \mathrm{~N}$

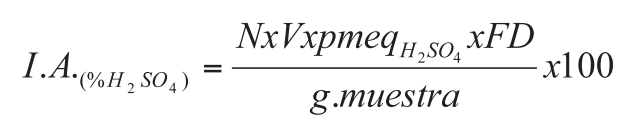

I.A. = Índice de acidez expresado en porcentaje de ácido sulfúrico

$\mathrm{N}=$ normalidad de la base

$\mathrm{V}=$ volumen de la base, $\mathrm{ml}$

$\mathrm{FD}=$ factor de dilución

$p$ meq = peso miliequivalente del ácido sulfúrico.

g. muestra = peso de muestra expresado, $g$

\section{Determinación de humedad}

Método: Pérdida por secado (Guía Lab. Análisis de Alimentos)

\section{Materiales y Equipos:}

Cajas de aluminio de $10 \mathrm{~cm}$. de diámetro de fondo plano y $2 \mathrm{~cm}$. de profundidad

Mortero y pistilo

Espátula

Desecador provisto con desecante adecuado

Pinza para cápsulas

Cápsulas de aluminio de fondo plano de $6 \mathrm{~cm}$. de diámetro y $1,5 \mathrm{~cm}$ de profundidad

Estufa regulada a $130^{\circ} \mathrm{C}$

Balanza analítica son sensibilidad a 0,1 mg

$$
\operatorname{Humedad}(\%)=W 1+W 2-\frac{W 1 x W 2}{100}
$$

$\mathrm{W} 1$ = Promedio de las humedades determinado en 15 $\mathrm{g}$ de muestra

$\mathrm{W} 2=$ Promedio de las humedades determinado en $5 \mathrm{~g}$ de muestra.

\section{Determinación de aw}

\section{Materiales y Equipos:}

Equipo medidor de actividad de agua

Soluciones patrón de concentración molal conocida

\section{Determinación de los tiempos de vida útil}

Para la determinación del tiempo de vida útil se consideró a todas las determinaciones realizadas en el pan, según su variabilidad; sólo se trabajó con las que se consideran parámetros de calidad para el producto, se aplicó el método matemático de correlación y se comparó entre la reacción de orden cero y la reacción de primer orden.

Para la ecuación de orden cero se tienen:

$$
Q_{f}=Q_{o}-k \cdot t_{v}
$$

Para la ecuación de primer orden se establece:

$$
\ln Q_{f}=\ln Q_{o}-k . t_{v}
$$

\section{Análisis sensorial del pan}

\section{Descripción de los jueces:}

El análisis sensorial se realizó los días lunes luego de ser horneado. Se contó con jueces no entrenados. 


\section{Procedimiento de degustación}

A cada degustador se le presentó una bandeja con cuatro panes que estuvieron rotulados utilizando una tabla de números aleatorios de tres dígitos, un vaso con agua fresca, un esfero y cuestionario; a cada juez se le ubicó en lugares separados y se le pidió que evaluara en el siguiente orden: color, olor, sabor, textura.

\section{Evaluación sensorial}

Las evaluaciones sensoriales que se llevaron a cabo fueron:

\section{Color \\ Olor \\ Sabor \\ Textura}

Los datos recopilados se transformaron de acuerdo a los valores asignados en la escala hedónica, la misma que tiene un rango de valores de 1 a 7 ; los mismos que son crecientes de acuerdo al gusto o disgusto del consumidor.

Se utilizó un diseño de bloques completamente al azar y para cuya interpretación se utiliza la prueba de Friedman en el análisis de varianza y las pruebas de significancia de Tukey al $5 \%$.

\section{Resultados y Discusión}

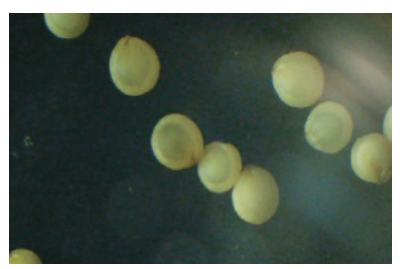

Vista al estereomicroscopio.

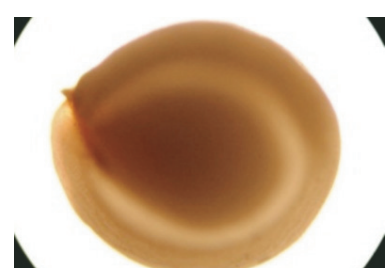

Vista al microscopio.

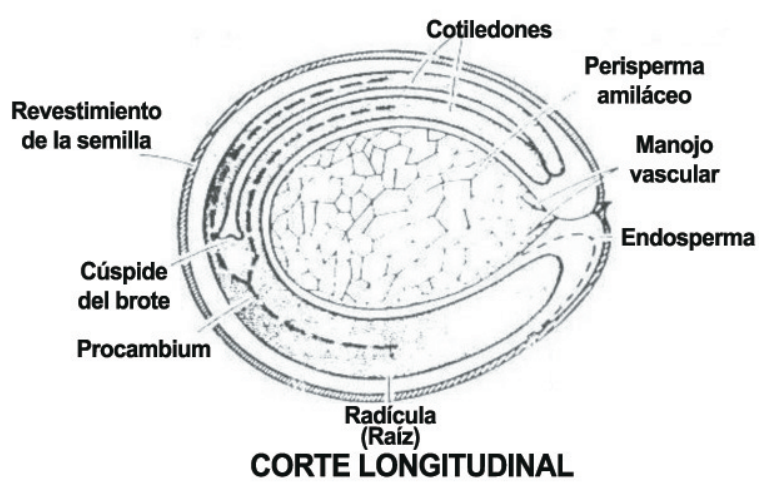

Figura 1. Semilla de amaranto
Tabla 1. Caracterización de harina de amaranto

\begin{tabular}{cc}
\hline DETERMINACIÓN & $\begin{array}{c}\text { HARINA DE SEMILLA DE } \\
\text { AMARANTO }\end{array}$ \\
\hline $\mathrm{pH}$ & 6,75 \\
Acidez $\left(\% \mathrm{H}_{2} \quad \mathrm{SO}_{4}\right)$ & 0,08 \\
Humedad \% & 10,00 \\
Grasa \% & 4,10 \\
Ceniza \% & 3,17 \\
Proteína \% & 12,49 \\
Fibra \% & 5,18 \\
Carbohidratos & 65,06 \\
Totales \% & 1,03 \\
Azucares Reductores & \\
$\%$ & 61,69 \\
Azúcares Totales\% & \\
Almidón \% & 57,63 \\
(mét. cálculo) & \\
Almidón \% & 58,97 \\
(mét. Ewers) &
\end{tabular}

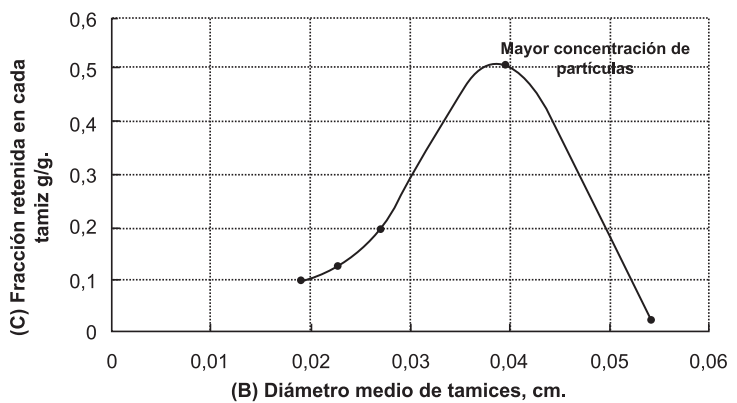

Figura 2. Análisis granulométrico: Diagrama diferencial

Tabla 2. Aminoácidos presentes en harina de amaranto

\begin{tabular}{|c|c|c|c|}
\hline AMINOÁCIDO & $\%$ & AMINOÁCIDO & $\%$ \\
\hline Ac. Aspártico & 1,17 & Metionina & 0,27 \\
\hline Treonina & 0,45 & Isoleucina & 0,46 \\
\hline Serina & 0,75 & Leucina & 0,69 \\
\hline Ac. Glutámico & 2,67 & Tirosina & 0,42 \\
\hline Prolina & 0,49 & Fenilalanina & 0,55 \\
\hline Glicina & 1,00 & Histidina & 0,36 \\
\hline Alanina & 0,49 & Lisina & 0,81 \\
\hline Cistina & 0,26 & Arginina & 1,11 \\
\hline Valina & 0,53 & Triptófano & 0,17 \\
\hline \multicolumn{4}{|c|}{ Determinación en base seca $(\mathrm{g} / 100 \mathrm{~g})$} \\
\hline \multicolumn{4}{|c|}{ Tabla 3. Características del almidón } \\
\hline \multicolumn{2}{|c|}{ DETERMINACIÓN } & \multicolumn{2}{|c|}{ RESULTADO } \\
\hline \multicolumn{2}{|c|}{ Color } & \multicolumn{2}{|c|}{ Blanco } \\
\hline \multicolumn{2}{|c|}{ Apariencia } & \multicolumn{2}{|c|}{ Polvo Homogénea } \\
\hline \multicolumn{2}{|c|}{ Morfología del gránulo } & \multicolumn{2}{|c|}{ Redondo } \\
\hline \multicolumn{2}{|c|}{$\begin{array}{l}\text { Diámetro medio de gránulos, } \\
\qquad \mu \mathrm{m}^{\star *}\end{array}$} & \multicolumn{2}{|c|}{0,9} \\
\hline \multicolumn{2}{|c|}{ Amilosa \% } & \multicolumn{2}{|c|}{$3,24^{*}$} \\
\hline \multicolumn{2}{|c|}{ Amilopectina \% } & \multicolumn{2}{|c|}{$96,76^{*}$} \\
\hline
\end{tabular}

* Determinación en base seca $\quad$ ** Por comparación 
Análisis realizados en pan

Tabla 4. Determinación de pH

\begin{tabular}{ccccccc}
\hline F.V. & G.L. & S.C. & C.M. & F.C. & $\begin{array}{r}\text { F.T. } \\
\mathbf{5 \%}\end{array}$ & $\begin{array}{c}\text { F.T. } \\
\mathbf{1 \%}\end{array}$ \\
\hline Total & 15 & 0,1571 & & & & \\
Bloques & 3 & 0,03195 & $0,0,01065$ & 0,8 & 3,86 & 6,99 \\
Tratamientos & 3 & 0,00565 & 0,00188 & 0,14 & 3,86 & 6,99 \\
E. Exp. & 9 & 0,1195 & 0,01328 & & & \\
\hline C. V. $=2 \%$ & & & & & &
\end{tabular}

Tabla 5. Determinación de acidez

\begin{tabular}{ccccccc}
\hline F.V. & G.L. & S.C. & C.M. & F.C. & $\begin{array}{r}\text { F.T. } \\
\mathbf{5 \%}\end{array}$ & $\begin{array}{c}\text { F.T. } \\
\mathbf{1 \%}\end{array}$ \\
\hline Total & 15 & & & & & \\
Bloques & 3 & 0,00004 & 0,00004 & 2,55 & 3,86 & 6,99 \\
Tratamientos & 3 & 0,00001 & 0,00001 & 0,6 & 3,86 & 6,99 \\
E. Exp. & 9 & 0,00005 & 0,00005 & & & \\
\hline C. V. $=4 \%$ & & & & &
\end{tabular}

Tabla 6. Determinación de humedad

\begin{tabular}{ccc}
\hline DÍAS & MEDIA & RANGO \\
\hline D1 & 23,81 & A \\
D2 & 21,32 & B \\
D3 & 18,64 & \multicolumn{2}{c}{ C } \\
D4 & 18,18 & \multicolumn{2}{c}{ C } \\
\hline
\end{tabular}

Prueba de Tukey al 5\%

Tabla 7. Determinación de actividad acuosa $a_{w}$

\begin{tabular}{ccc}
\hline TRATAMIENTO & MEDIA & RANGO \\
\hline T4 & 0,808 & A \\
T1 & 0,803 & A B \\
T3 & 0,797 & \multicolumn{2}{c}{ B } \\
T2 & 0,776 & \multicolumn{2}{c}{ C } \\
\hline
\end{tabular}

Prueba de Tukey al 5\%

Tabla 8. Tiempo de vida útil de los productos

\begin{tabular}{ccc}
\hline \multirow{2}{*}{ Formulación } & \multicolumn{2}{c}{ tv (días) } \\
& Orden cero & Primer orden \\
\hline $0 \%$ & 2,00 & 1,95 \\
$5 \%$ & 2,22 & 2,14 \\
$10 \%$ & 2,09 & 2,03 \\
$15 \%$ & 1,97 & 1,92 \\
\hline
\end{tabular}

Tabla 9. Evaluación de color

\begin{tabular}{c|c|c}
\hline TRATAMIENTO & MEDIA & RANGO \\
\hline T1 & 6,12 & A \\
T2 & 5,71 & A B \\
T3 & 5,18 & A B \\
T4 & 5 & B \\
\hline
\end{tabular}

12. Evaluación de olor

\begin{tabular}{ccc}
\hline TRATAMIENTO & MEDIA & RANGO \\
\hline T1 & 6,24 & A \\
T2 & 5,47 & A \\
T3 & 5 & B \\
T4 & 5 & B \\
\hline
\end{tabular}

Tabla 10. Evaluación de sabor

\begin{tabular}{cccccc}
\hline F.V. & G.L. & S.C. & C.M. & F.C. & $\begin{array}{c}\text { F.T. } \\
\mathbf{5 \%}\end{array}$ \\
\hline Total & 67 & 61,06 & & & \\
Tratamientos & 3 & 3,3 & 1,1 & 1,24 & 2,84 \\
Jueces & 16 & 15,06 & 0,94 & 1,06 & 1,92 \\
Error & 48 & 42,7 & 0,89 & & \\
\hline
\end{tabular}

14. Evaluación de textura

\begin{tabular}{|c|c|c|}
\hline TRATAMIENTO & MEDIA & RANGO \\
\hline T1 & 6,24 & A \\
\hline $\mathrm{T} 2$ & 6 & $A \quad B$ \\
\hline T3 & 5,47 & A B \\
\hline T4 & 5,18 & B \\
\hline
\end{tabular}

\section{Conclusiones}

\subsection{Análisis físico - químicos de la harina}

La harina de semilla de amaranto Amaranthus caudatus elaborada utilizando un molino de disco sencillo debe pasar por una malla 32 equivalente a $0,495 \mathrm{~mm}$ de diámetro en un porcentaje de $97 \%$.

La harina de amaranto obtenida es de apariencia homogénea y finura ligeramente granulosa, su color ligeramente pardo, olor y sabor característicos.

El principal componente de la harina son los carbohidratos; de los que aproximadamente el $88 \%$ corresponden al almidón. El almidón está integrado por $96 \%$ de amilopectina, de gránulos redondos, diámetro de 0,9 um y su máxima gelificación a $55^{\circ} \mathrm{C}$ con 510 U.B.

Por el contenido de fibra presente es una harina integral ya que posee niveles iguales y superiores al de las harinas integrales.

El mayor contenido de grasa en comparación a las harinas obtenidas de los cereales tradicionales incrementa el valor energético del producto.

\subsection{Análisis físico - químicos del pan}

Al evaluar entre sí los valores de $\mathrm{pH}$ y acidez de las cuatro formulaciones de pan elaboradas, se determina 
que no existe diferencia entre ellas y los valores oscilan alrededor de una media de 5,60 para el $\mathrm{pH}$ y de 0,0675\% para la acidez, expresado en ácido sulfúrico. Se concluye por tanto que $\mathrm{pH}$ y acidez no son parámetros de calidad para la determinación del tiempo de vida útil de los productos elaborados.

A diferencia de las determinaciones de $\mathrm{pH}$ y acidez, la humedad no oscila alrededor de un valor medio sino que desciende en función del tiempo, por lo que es el parámetro de calidad que define el tiempo de vida de los productos elaborados.

La evaluación de la actividad de agua en las formulaciones analizadas confirma que existe mayor disponibilidad de agua durante los primeros días analizados y desciende a medida que la humedad disminuye; pero, no se observa relación de tipo lineal por lo que sus resultados no pueden extrapolarse.

El análisis de volumen manifiesta la diferencia de tamaño entre las formulaciones evaluadas. A menor concentración de harina de semilla de amaranto mayor volumen del pan.

El valor promedio de la densidad de los panes elaborados es de $0,249 \mathrm{~g} / \mathrm{ml}$, lo que indica que el tiempo de amasado fue normal y no intensificado.

Los panes elaborados poseen mayor cantidad de miga en relación a la corteza.

\subsection{Tiempo de vida útil y análisis sensorial del pan}

El color de los panes de las formulaciones del $0 \%, 5 \%$ y $10 \%$ son iguales entre sí y entre las formulaciones de los panes del $5 \%, 10 \%$ y $15 \%$, no se visualiza diferencia alguna.

La evaluación respecto al olor revela que el consumidor no logra diferenciar entre el blanco y la formulación del 5\%; así como tampoco logra diferenciar entre las formulaciones del $5 \%, 10 \%$ y $15 \%$ elaboradas con harina de amaranto. El olor que presentan las formulaciones del $10 \%$ y $15 \%$ es francamente distinto al olor del blanco. El sabor de todos los panes es igual y no hay diferencia entre ellos.

El consumidor no logra distinguir entre la textura del blanco y las formulaciones del $5 \%$ y $10 \%$; la textura de las formulaciones del $5 \%, 10 \%$ y $15 \%$ son iguales. La textura de los panes preparados con $15 \%$ de harina de amaranto difieren notablemente del blanco.

En virtud de la presente investigación, se acepta la hipótesis alterna; la harina se semilla de amaranto es factible de ser utilizada en la elaboración de pan en mezclas con harina de trigo.

Las cantidades de $5 \%, 10 \%$ y $15 \%$ utilizadas de harina de amaranto para la elaboración de pan no afectan el desarrollo del gluten, leudado, así como tampoco la formación de la miga.

\section{Referencias}

1. Anzaldua Morales, Antonio. La evaluación sensorial de los alimentos en la teoría y la práctica. Zaragoza: Acribia. 1990.

2. Badui Dergal Salvador. Química de los alimentos. 3 ed. México: Pearson educación. 1993.

3. Helmant, José. Farmacotecnia teórica y práctica. México: Continental. 1981. t IV.

4. Kirk, Ronal. Composición y análisis de alimentos de pearson. 2 ed. México: Continental. 1999.

5. Youfera, Primo. Química de los alimentos. Madrid: Síntesis. 2003.

5.2. Revistas:

6. Becker, Robert y Sauders, Robert. El amaranto: su morfología composición y usos como alimento y forraje. En: El amaranto y su potencial. Boletín Nº1 (marzo 1984).

7. Cavagnaro, J y Jain, S. Breve informe de una serie de estudios del amaranto de semilla. En: El amaranto y su potencial. Boletín No 3 (septiembre 1985).

8. Early, Daniel y Capistran de Early, Julia. Transferencia de tecnología indígena para la preparación de kiwicha (Amaranthus) primera parte. En: El amaranto y su potencial. Boletín Nº4 (diciembre 1987).

9. Monteros, Cecilia. Primera variedad mejorada de amaranto para la Sierra ecuatoriana. En: Boletín divulgativo. № 246 (abril 1994 ).

10. Pacheco de Delahaye, Emperatriz. Efecto de la temperatura sobre las propiedades funcionales de la harina de semilla de amaranto En: El amaranto y su potencial. Boletín № 1 (marzo 1987).

11. Vietmeyer, Noel. Cultivos incas, alimentos redescubiertos. En: Selecciones. (Agosto 1987).

12. Villegas, Evangelina. Determinación de triptófano. En: Métodos químicos usados en el centro internacional de mejoramiento de maíz y trigo para determinar la calidad de proteína de los cereales. 1985.

Otras Fuentes:

13. Morán, Marco. Análisis del pan. En: Prácticas de laboratorio de análisis de alimentos. Quito: Facultad de Ciencias Químicas.

14. Morán, Marco. Determinación de almidón. En: Prácticas de laboratorio de análisis de alimentos. Quito: Facultad de Ciencias Químicas.

15. Morán, Marco. Determinación de fibra cruda. En: Prácticas de laboratorio de análisis de alimentos. Quito: Facultad de Ciencias Químicas.

16. Norma INEN 95. Pan común requisitos. 1979-06.

17. Norma INEN 521. Harina de origen vegetal. Determinación de la acidez titulable.1980-12.

18. Norma INEN 526. Harina de origen vegetal. Determinación de la concentración de iones hidrógeno. $1980-12$.

19. Norma INEN 616. Harina de trigo requisitos. Segunda revisión. 1998 - 03 